\title{
Imp3 Expression in Benign and Malignant Thyroid Tumors and Hyperplastic Nodules
}

\author{
Sezer Kulaçoğlu, Gamze Erkılınç
}

Department of Pathology, Ankara Numune Training and Research Hospital, Ankara, Turkey

Background: IMP3, a member of insulin-like growth factor II $\mathrm{m}$ RNA binding protein family, seems to be promising in the diagnosis of carcinomas of many organs as well as malignant melanomas and some sarcomas. It is postulated that it might be a marker of malignancy. The results of the few prior studies indicate that IMP3 has the potential to be useful in distinguishing benign and malignant tumors of thyroid.

Aims: We aimed to evaluate the immunohistochemical expression of IMP3 in non-neoplastic nodules and benign and malignant tumors of the thyroid.

Study Design: Diagnostic accuracy study.

Methods: Overall, 92 thyroid lesions, including 22 nodular hyperplasia (NH), 14 follicular adenoma (FA), 9 follicular carcinoma (FC), 37 papillary carcinoma (PC) (15 follicular variant), 3 well differentiated carcinoma-not otherwise specified (WDC-NOS), 4 poorly differentiated carcinoma (PDC) and anaplastic carcinoma (AC) were included. Immunohistochemically, cytoplasmic expression of IMP3 was evaluated in terms of extent and intensity of the staining semi-quantitatively and an immunohistochemical score (IHS) was obtained for each case. A score higher than 2 was considered positive staining.

Results: In contrast with previous studies, we observed positive staining in benign lesions, especially in benign tumors. For identifying malignant tumors, the sensitivity of IMP3 was $82.1 \%$, specificity was $33.3 \%$, positive predictive value (PPV) was $65.7 \%$ and negative predictive value (NPV) was 54.5\%. In distinguishing neoplastic and hyperplastic lesions, the sensitivity was $50 \%$, specificity was $15.7 \%$, PPV was $15.7 \%$ and NPV was $50 \%$. The IMP3 expression was similar for FA and well differentiated carcinomas $(\mathrm{p}=0.434)$, but there was a significant difference between hyperplastic nodules and FA $(\mathrm{p}=0.011)$.

Conclusion: Our data suggest that IMP3 is effective in discriminating hyperplastic and neoplastic lesions but not useful in differentiating benign tumors from malignant tumors.

Keywords: Immunohistochemistry, IMP3, thyroid, tumor
The diagnosis of malignancy mostly rests on morphological features in thyroid pathology. Although immunohistochemistry has been proven to be a very efficient and useful technique in the routine practice of a pathologist, its role in the differential diagnosis of the thyroid nodules is still restricted. The most useful area is distinguishing follicular cell derived tumors from other lesions like $\mathrm{C}$ cell-derived endocrine tumors and rarely from metastatic tumors.
On the other hand, the differential diagnosis of follicular cell derived hyperplastic and neoplastic lesions may be very problematic and the issue is seldom solved by immunohistochemistry.

The well-known diagnostic challenge of thyroid pathology is the differential diagnosis of follicular adenoma (FA) and minimally invasive follicular carcinoma (MIFC) (1). The distinction of these lesions depends on the presence of capsule and/or ves- 


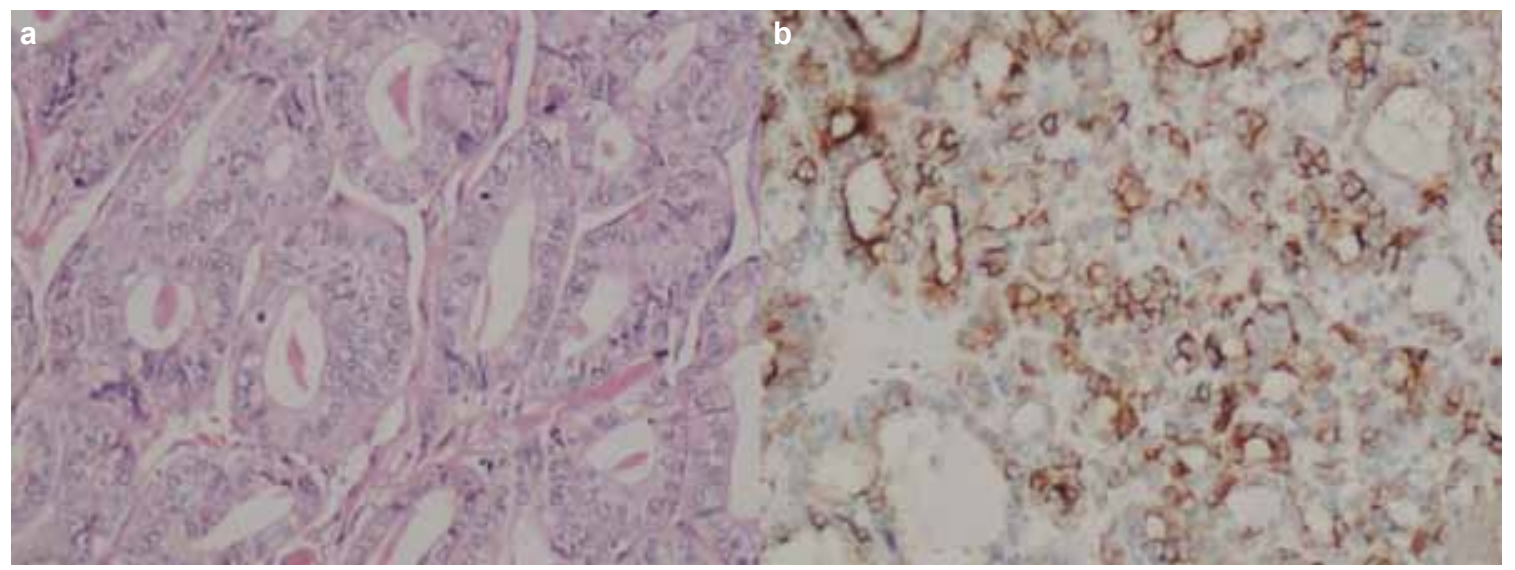

FIG. 1. a, b. A case reported as papillary carcinoma follicular variant, H\&E x200 (a). HBME-1 expression in papillary carcinoma follicular variant, x200 (b)

sel invasion which can sometimes be very difficult to demonstrate, despite thorough sampling and proper sectioning.

However, recently, the encapsulated follicular patterned nodules showing diffuse or focally intermediate nuclear features of papillary carcinoma have become another source of confliction (2). If the lesion shows capsular and/or vascular invasion, it is then possible to make a diagnosis of well differentiated carcinoma without further subtyping. However, still this might not be adequate for clinicians. If there is no invasion, the differential diagnosis has to be made between follicular adenoma (FA), a benign neoplasm, and papillary carcinoma follicular variant (PCFV) - a malignant tumor. This critical decision cannot be made morphologically and even immunohistochemically for some nodules; these cases are reported as 'well differentiated tumor of uncertain malignant potential' (WDTUMP) (2).

The most widely used immunohistochemical markers to solve this problem are CK19, HBME1 and galectin $3(1,2)$. These antibodies can be helpful, especially when used together, but studies have shown that each has some disadvantages and limitations (1-6). More reliable markers are needed to distinguish benign and malignant thyroid neoplasms.

Recently, IMP3, a member of the 'insulin-like growth factor II mRNA binding protein' family, has been presented as a potential diagnostic and prognostic tool for thyroid tumors (7-9).

IMP3 affects cell proliferation, and has been suggested to display vital function in RNA stabilization, cell growth and migration during embryogenesis (10-12). It is postulated that IMP3 is expressed at low or undetectable levels in mature tissues, except for the placenta (8). However, the expression of IMP3 was reported in tumors of many organs such as the kidney (13), liver (14), endometrium (15), cervix (16), ovary (17), breast (18-19), pancreas (20), stomach (21), colon (22), bladder (23), and lung (24), as well as in malignant melanoma (25) and some sarcomas (26-27). Since IMP3 is detected in fetal and neonatal tissues and various tumors, it is considered an oncofetal protein. IMP3 is also known to be present in stem cells (28).

Depending on the fact that IMP3 is detected in different cancer types at high levels, it can be postulated that IMP3 might have a role in carcinogenesis, the proliferation of malignant cells and tumor progression.

Some prior studies have investigated the usefulness of IMP3 expression in benign and malignant thyroid tumors, either by immunohistochemistry or by using the qRT-PCR technique. The authors concluded that IMP3 can be useful in distinguishing benign and malignant thyroid tumors (7-9).

The aim of this study is to evaluate the diagnostic utility of IMP3 in differentiating benign and malignant tumors and in separating neoplastic and hyperplastic nodules of the thyroid by using immunohistochemistry.

\section{MATERIALS AND METHODS}

This study protocol was approved by the local ethic committee.

\section{Specimen}

A total of 92 cases, including 22 nodular hyperplasia (NH), 14 FA (6 oncocytic variant), 22 conventional papillary carcinoma (CPC), 15 PCFV, 9 MIFC, 3 well differentiated carcinoma-not otherwise specified (WDC-NOS), 4 poorly differentiated carcinoma (PDC), and 3 anaplastic carcinoma (AC) were identified between 2007 and 2013.

The H\&E stained slides were reviewed by two observers simultaneously. Immunohistochemical (IHC) techniques (galectin3, HBME-1, CK19) had already been used in most of the follicular patterned tumors. The diagnosis was confirmed by evaluating both $\mathrm{H} \& \mathrm{E}$ stained and immunostained slides (Figure 1a, b). A representative block was selected for IHC from each case. The remarkable microscopic features such as 
the presence of capsule and vessel invasion, extrathyroidal invasion or lymph node metastasis were noted.

Patients' demographics and macroscopic features (the size of the thyroid and the nodule, multifocality, laterality) were obtained from the original report and compared with the microscopic findings.

\section{Immunohistochemical technique and evaluation}

Three micron sections were obtained from formalin-fixed paraffin-embedded tissues and the slides were stained using the IMP3 antibody (orb40603, rabbit polyclonal, Biorbyt, Cambridge, UK), dilution 1:200, in the autostainer (Bond; Leica Biosystems, Newcastle, UK) at room temperature.

Normal thyroid tissue adjacent to the hyperplastic nodule or tumors was present in almost every case as a negative control.

Cytoplasmic staining was considered positive and graded according to the extent and intensity of the staining by two observers at the same time.

According to the extent of the staining:

Score 0: None of the cells are positive in the lesion.

Score $1:<10 \%$ cells express cytoplasmic positivity.

Score $2: 10-50 \%$ of the cells express cytoplasmic positivity.

Score $3:>50 \%$ cells express cytoplasmic positivity.

The intensity of the staining was evaluated as no staining (score 0 ), weak (score 1), moderate (score 2) and strong (score 3).

An immunohistochemical score (IHC-score) was obtained by adding two scores (the lowest IHC score was 0 and the highest IHC score was 6); a score higher than 2 was considered positive, whereas a score 2 or lower was considered negative (9).

We also compared the median IHC score of the groups.

\section{Statistical analysis}

Data were analysed using the Statistical Package 15.0 for Windows (SPSS Inc., Chicago, IL, USA). The normality of variables was calculated via Kolmogorov-Smirnov test or Shapiro-Wilk test. Double comparisons between non-normally distributed variables were performed by the Mann Whitney-U test and multiple comparisons between non-normally distributed variables were performed with the Kruskal Wallis test. Post-hoc comparisons with Bonferroni correction were used to detect the source of the difference between the groups. Chi squared was used for categorical variables. Fisher's exact test and Fisher Freeman Halton test were used when the expected values in any of the cells of a contingency table were below 5. Statistically significant importance was considered for $\mathrm{p}<0.05$.

\section{RESULTS}

The age of the patients ranged from 18 years to 80 years; the mean age was $46.7 \pm 13.7$. Mean age for the malignant tumors and for benign lesions were 47.7 and 46.2 , respectively, and was not statistically significant $(\mathrm{p}=0.627)$.

The mean age for $\mathrm{PC}$ was $42 \pm 3.7$, for PCFV was $49.4 \pm 10.7$, for MIFC was $42.2 \pm 48$, for PDC was $52.7 \pm 9.2$ and for AC was $68.6 \pm 12$. The mean age for AC was statistically significant between these groups $(\mathrm{p}=0.02)$. There was a female predilection for both benign and malignant lesions. Of the 92 cases, $72(78.9 \%)$ were female. Overall, 56 cases had malignant tumors, 43 of whom were female $(76.8 \%)$, and 36 cases had benign lesions, 29 of whom were female $(80.6 \%)$. The mean diameter for malignant lesions was $2.5 \mathrm{~cm}$ and for benign lesions was 2.97. The mean diameters of the benign and malignant lesions were not significantly different $(\mathrm{p}=0.051)$.

When all of the cases were evaluated in terms of localization, there was a predilection for the right lobe. For CPC, 53\% of the tumors were in the right lobe, $20 \%$ in the left lobe and $26.7 \%$ of cases were bilateral. PCFV was also mostly (45.5\%) located on the right lobe, $27.3 \%$ were located on the left lobe and $27.3 \%$ of the tumors were bilateral. FA was distributed equally: $50 \%$ on the right lobe, $50 \%$ on the left lobe. Interestingly, $88.9 \%$ of the MIFC was located on the right lobe. NH was $81.8 \%$ bilateral.

When the capsule invasion was compared between the subtypes of the carcinomas, capsule invasion, as expected by definition, was observed in $100 \%$ of MIFC and WDC-NOS. None of the CPC cases were encapsulated, whereas $25 \%$ of the PCFV cases was encapsulated but none of them showed capsule invasion. On the other hand, $25 \%$ of the PDC cases were encapsulated and all showed capsule invasion.

Of the malignant tumors, $21.4 \%$ showed lymphatic/vascular invasion. Also, $100 \%$ of AC, $75 \%$ of PDC, $33.3 \%$ of MIFC and $13.6 \%$ of CPC showed vessel invasion. We did not observe vessel invasion in any case of PCFV and WDC-NOS.

The presence of extrathyroidal invasion (ETI) and metastatic lymph node (MLN) status in malignant tumors were evaluated. Overall, 9 out of 22 cases of CPC showed ETI; 6 of them were MLN-positive. In other words, all CPC cases with MLN showed ETI.

\section{IMP3 staining}

The results of the evaluation of the IMP3 staining are shown in Table 1.

We observed positive staining (higher than IHC-score 2) in $50 \%$ (11 cases) of the NH (Figure 2a, b). None of the NH cases had an IHC score of 5 or 6 . All but one of the 13 (92.9\%) cases of FA showed positivity (Figure 3a, b). There was a statistically significant difference between $\mathrm{NH}$ and FA cases $(\mathrm{p}=0.011)$ (Table 2).

Benign lesions (sum of NH and FA cases) showed $66.7 \%$ and malignant tumors showed $82.1 \%$ positivity for IMP3. There was no significant difference between the two groups statisti- 


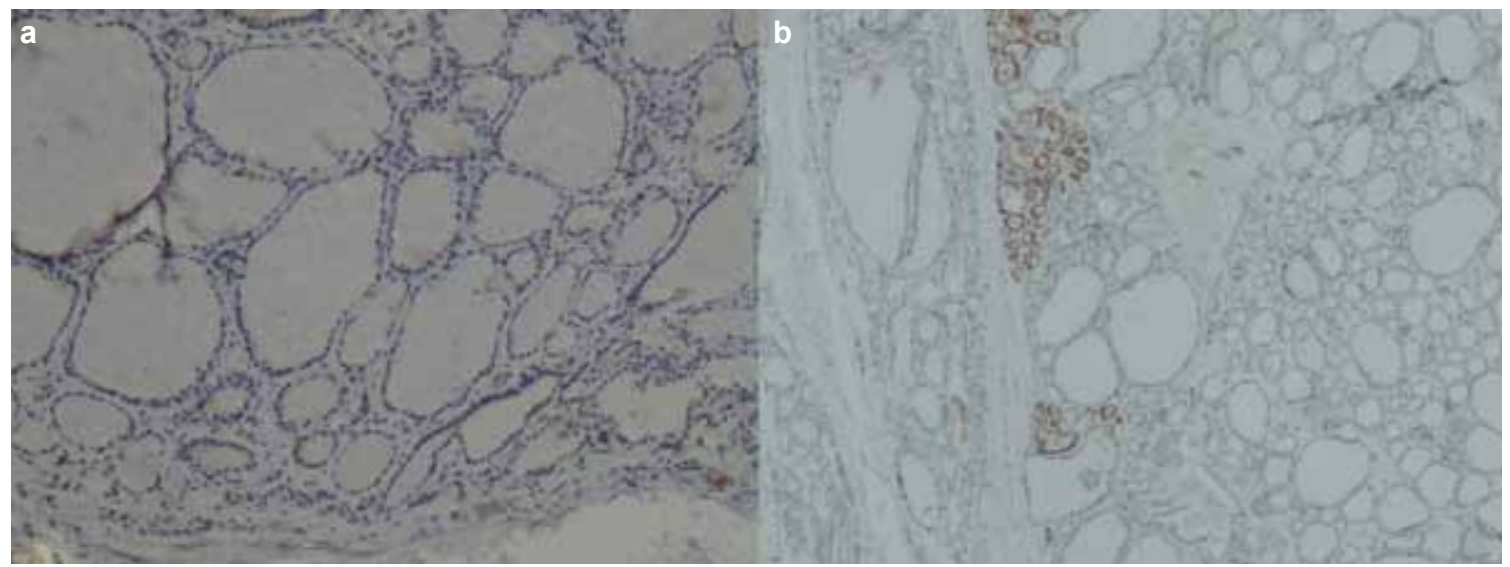

FIG. 2. a, b. Nodular hyperplasia with negative IMP3 staining, $x 100$ (a). Nodular hyperplasia with IMP3 staining. The staining was less than $10 \%$ of the cells but strong (IHC score 4). Note that the adjacent normal thyroid tissue is negative for IMP3, x40 (b)

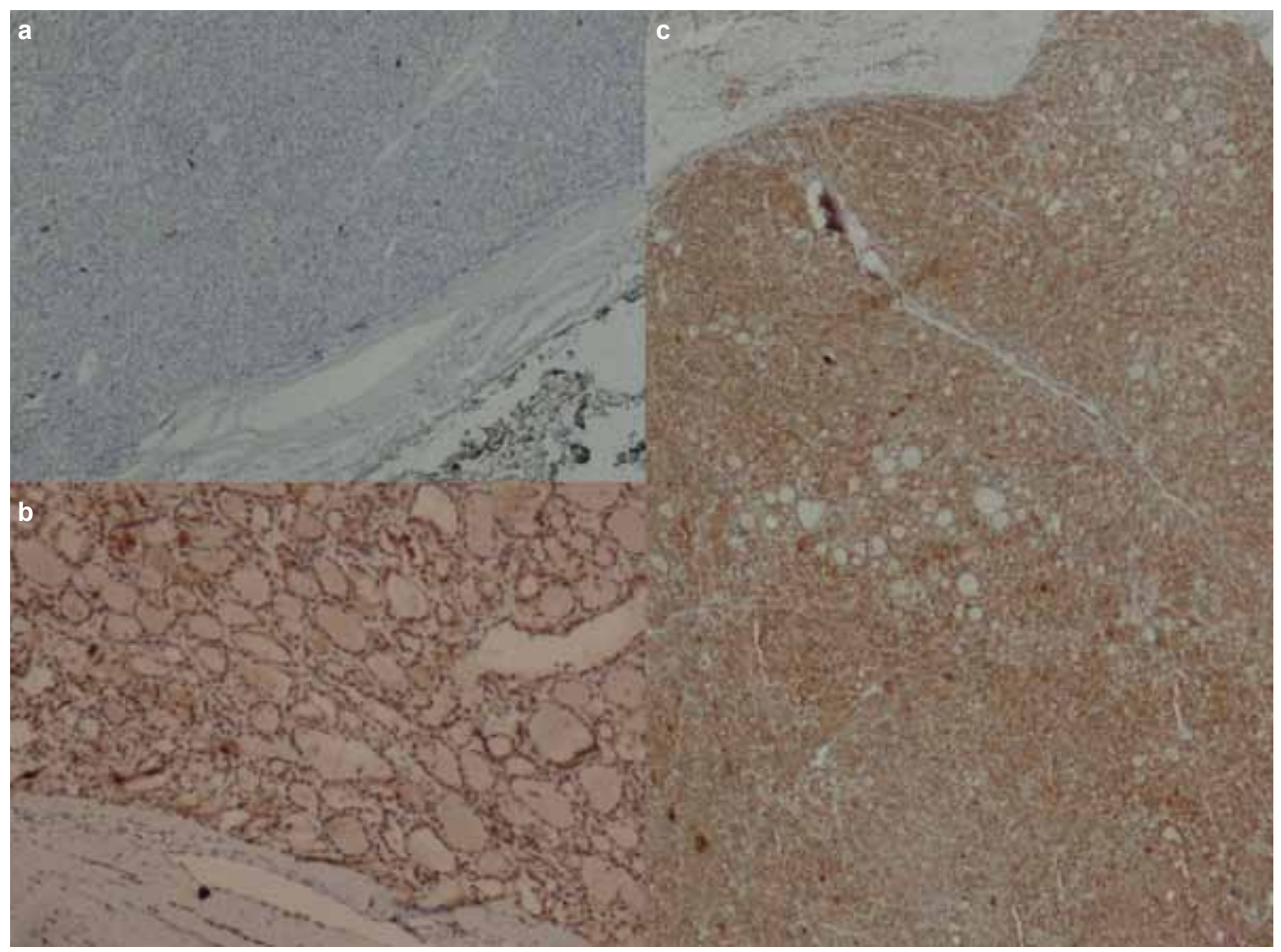

FIG. 3. a-c. Follicular adenoma with negative IMP3 staining, $x 40$ (a). Follicular adenoma with IMP3 staining extensively with moderate intensity (IHC-score 5), x100 (b). Minimally invasive follicular carcinoma with extensive and strong IMP3 positivity (IHC-score 6), x40 (c)

cally $(\mathrm{p}=0.132)$ (Table 3$)$. Benign tumors (FA) and well differentiated carcinomas were compared and no significant difference between the groups was found statistically $(\mathrm{p}=0.434)$. When malignant tumors were compared to each other, there was no significant difference between the groups $(p=0.537)$ (Table 4) (Figure $3 c$ and Figure $4 a-d)$. We compared the staining of the follicular patterned neoplastic lesions to each other, but no significant difference was found $(\mathrm{p}=0.247)$. Also, when CPC and PCFV were compared, there was no significant difference $(\mathrm{p}=0.408)$.

For malignant tumors, the sensitivity of IMP3 was $82.1 \%$, specificity was $33.3 \%$, positive predictive value (PPV) was 
TABLE 1. The results of the evaluation of the IMP3 staining

\begin{tabular}{|c|c|c|c|c|c|c|c|c|}
\hline \multirow[b]{2}{*}{ IHC-score } & \multicolumn{3}{|c|}{ Negative } & \multicolumn{4}{|c|}{ Positive } & \multirow[b]{2}{*}{ Median IHC-score } \\
\hline & 0 & 1 & 2 & 3 & 4 & 5 & 6 & \\
\hline $\mathrm{NH}, \mathrm{n}(\%)$ & $7(31.8)$ & $0(0)$ & $4(18.2)$ & $7(31.8)$ & $4(18.2)$ & $0(0)$ & $0(0)$ & $2.5(\min : 0, \max : 4)$ \\
\hline $\mathrm{FA}, \mathrm{n}(\%)$ & $0(0)$ & $0(0)$ & $1(7.1)$ & $2(14.3)$ & $2(14.3)$ & $4(28.5)$ & $5(35.8)$ & 5 (min: $2, \max : 6)$ \\
\hline CPC, n (\%) & $0(0)$ & $0(0)$ & $3(13.6)$ & $3(13.6)$ & $5(22.8)$ & $7(31.8)$ & $4(18.2)$ & $4.5(\min : 2, \max : 6)$ \\
\hline PCFV, n (\%) & $0(0)$ & $0(0)$ & $4(26.6)$ & $2(13.3)$ & $6(40.2)$ & $2(13.3)$ & $1(6.6)$ & 4 (min: $2, \max : 6)$ \\
\hline WDCNOS, n (\%) & $0(0)$ & $0(0)$ & $0(0)$ & $0(0)$ & $0(0)$ & $0(0)$ & $3(100)$ & 6 (min: $6, \max : 6)$ \\
\hline MIFC, n (\%) & $0(0)$ & $0(0)$ & $2(22.2)$ & $1(11.1)$ & $1(11.1)$ & $2(22.2)$ & $3(33.4)$ & 5 (min: $2, \max : 6)$ \\
\hline PDC, n (\%) & $0(0)$ & $0(0)$ & $0(0)$ & $0(0)$ & $2(66.6)$ & $0(0)$ & $1(33.4)$ & 5 (min: 4, max: 6) \\
\hline $\mathrm{AC}, \mathrm{n}(\%)$ & $0(0)$ & $0(0)$ & $1(33.3)$ & $0(0)$ & $0(0)$ & $1(33.3)$ & $1(33.3)$ & $5(\min : 2, \max : 6)$ \\
\hline
\end{tabular}

NH: nodular hyperplasia; FA: follicular adenoma; CPC: conventional papillary carcinoma; PCFV: papillary carcinoma follicular variant; WDCNOS: well differentiated carcinoma-not otherwise specified; MIFC: minimally invasive follicular carcinoma; PDC: poorly differentiated carcinoma; AC: anaplastic carcinoma; min: minimum; max: maximum

TABLE 2. IMP3 expression in benign lesions

\begin{tabular}{lccc} 
& Negative & Positive & Total \\
\hline NH, n (\%) & $11(50)$ & $11(50)$ & $22(100)$ \\
FA, n (\%) & $1(7.1)$ & $13(92.9)$ & $14(100)$ \\
& $\mathrm{p}=0.011$ & & \\
\hline
\end{tabular}

NH: nodular hyperplasia; FA: follicular adenoma

TABLE 3. Comparison of the IMP3 expression in benign lesions and malignant tumors

\begin{tabular}{lccc}
\hline & Negative & Positive & Total \\
\hline Benign lesions, (including NH), n (\%) & $12(33.3)$ & $24(66.7)$ & $36(100)$ \\
Malignant tumors, n (\%) & $10(17.9)$ & $46(82.1)$ & $56(100)$ \\
& $\mathrm{p}=0.132$ & & \\
\hline
\end{tabular}

NH: nodular hyperplasia

$65.7 \%$ and negative predictive value (NPV) was $54.5 \%$. In distinguishing neoplastic and hyperplastic lesions, the sensitivity was $50 \%$, specificity was $15.7 \%$, PPV was $15.7 \%$ and NPV was $50 \%$. When the value of IMP3 for identifying PC was evaluated, the sensitivity of IMP3 for PC was detected to be $81.1 \%$ and specificity was $27.3 \%$. The PPV was $42.9 \%$ and the NPV was $68.2 \%$.

We also compared the median IHC score of the groups:

Statistically, there was a significant difference between $\mathrm{NH}$ and FA ( $\mathrm{p}=0.000)$, and between $\mathrm{NH}$ and all neoplastic lesions $(\mathrm{p}=0.001)$. There was no significant difference between benign (FA) and malignant tumors $(\mathrm{p}=0.265)$ and between FA and well differentiated carcinomas $(\mathrm{p}=0.204)$. Also, no significant difference was found when the malignant tumors were compared each other $(\mathrm{p}=0.106)$.

There were 5 metastatic (lymph nodes) papillary carcinomas; we performed IMP3 staining for both the primary tumor and the metastatic node and obtained similar staining patterns for primary and metastasis. Three of the primary tumors were positive for IMP3 and the metastases of these cases were also
TABLE 4. IMP3 expression in malignant tumors

\begin{tabular}{lccc}
\hline & Negative & Positive & Total \\
\hline CPC, n (\%) & $3(13.6)$ & $19(86.4)$ & $22(100)$ \\
PCFV, n (\%) & $4(26.7)$ & $11(73.3)$ & $15(100)$ \\
MIFC, n (\%) & $2(22.2)$ & $7(77.8)$ & $9(100)$ \\
WDCNOS, n (\%) & $0(0)$ & $3(100)$ & $3(100)$ \\
PDC, n (\%) & $0(0)$ & $4(100)$ & $4(100)$ \\
AC, n (\%) & $1(33.3)$ & $2(66.7)$ & $3(100)$ \\
& $\mathrm{p}=0.505$ & & \\
\hline
\end{tabular}

PC: papillary carcinoma; CPC: conventional papillary carcinoma; PCFV: papillary carcinoma follicular variant; MIFC: minimally invasive follicular carcinoma; WDCNOS: well differentiated carcinoma-not otherwise specified; PDC: poorly differentiated carcinoma; AC: anaplastic carcinoma

positive while the metastatic deposits of the negative primaries were negative.

We wanted to evaluate the relationship between IMP3 expression and pathological features of the malignant tumors. In applicable cases, there was no correlation between IMP3 expression and the macroscopic and histopathological features of the tumors (Table 5).

\section{DISCUSSION}

In this study, we aimed to evaluate the usefulness of IMP3 in differentiating benign and well differentiated malignant tumors of the thyroid. In routine practice, the most challenging area in thyroid pathology is the differential diagnosis of the follicular patterned encapsulated nodular lesions. In these situations, even the most experienced pathologists sometimes find difficulties in making differential diagnosis between benign and malignant lesions. For this reason, 2 borderline categories have been used for reporting these cases as 'well- differentiated follicular tumor of uncertain malignant potential' 


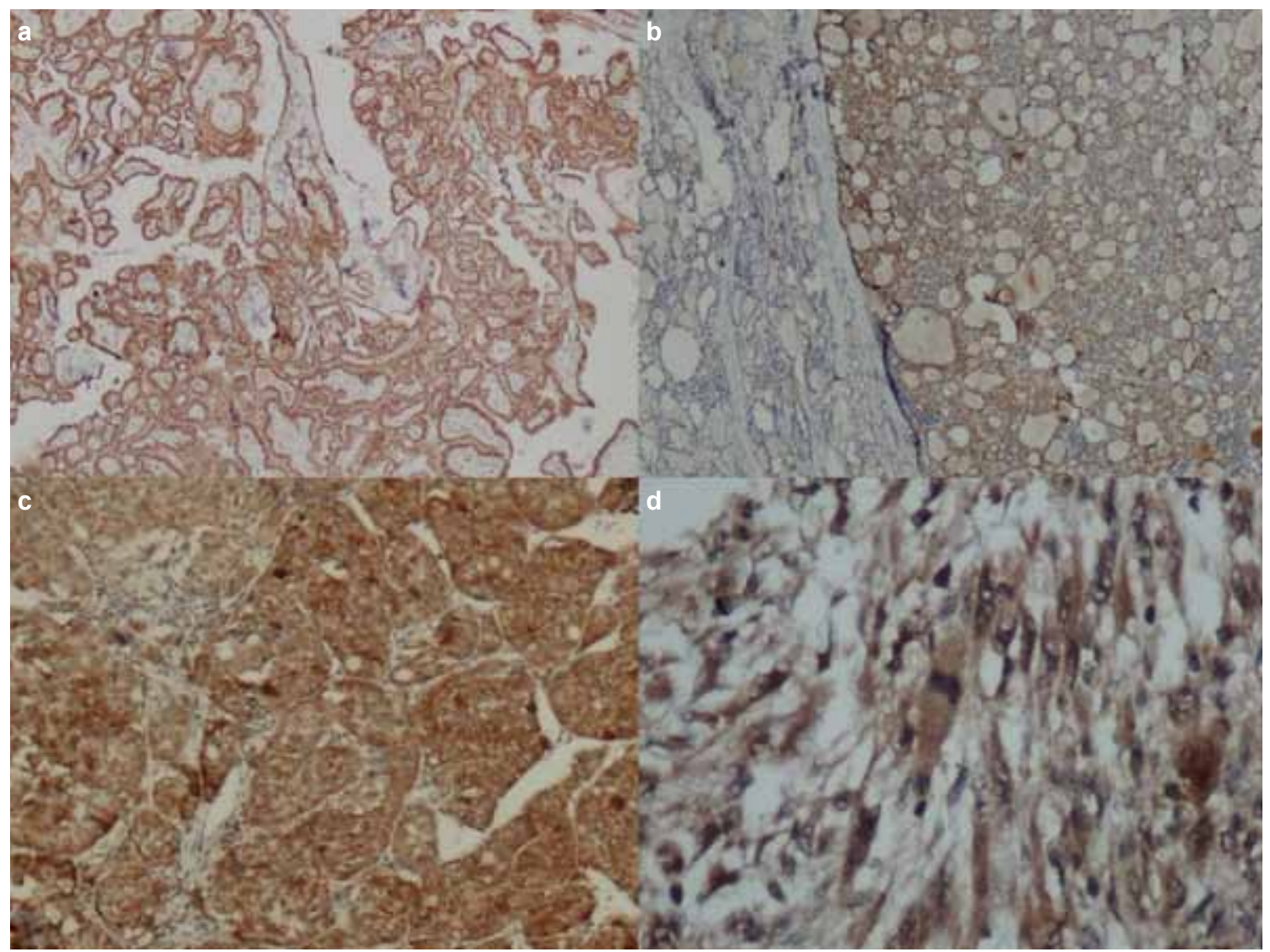

FIG. 4. a-d. Papillary carcinoma showing extensive and strong staining with IMP3 (IHC-score 6), x40 (a). Papillary carcinoma encapsulated follicular variant with extensive and moderately intensive staining (IHC-score 5). The normal thyroid tissue is negative, $x 40$ (b). Poorly differentiated carcinoma expressing IMP3 diffusely and strongly (IHC-score 6), x100 (c). Anaplastic carcinoma with extensive and strong IMP3 positivity (IHCscore 6), $x 400(d)$

TABLE 5. IMP3 expression and the pathological features of the malignant (well-differentiated) tumors*

\begin{tabular}{|c|c|c|c|c|c|c|}
\hline & \multicolumn{2}{|c|}{ CPC (n:22) } & \multicolumn{2}{|c|}{ PCFV (n:15) } & \multicolumn{2}{|c|}{ MIFC (n:9) } \\
\hline & IMP3- & IMP3+ & IMP3- & IMP3+ & IMP3- & IMP3+ \\
\hline Size, $\mathrm{cm} 1>$ & 0 & 7 & 1 & 5 & 0 & 1 \\
\hline $1-5$ & 3 & 11 & 2 & 6 & 2 & 6 \\
\hline $5<$ & 0 & 1 & 1 & 0 & 0 & 0 \\
\hline $\mathrm{p}$ value & \multicolumn{2}{|c|}{0.359} & \multicolumn{2}{|c|}{0.215} & \multicolumn{2}{|c|}{1.000} \\
\hline EI present (n) & 2 & 7 & 0 & 0 & 0 & 0 \\
\hline absent (n) & 1 & 12 & 4 & 11 & 2 & 7 \\
\hline $\mathrm{p}$ value & \multicolumn{2}{|c|}{1.000} & \multicolumn{2}{|c|}{ NA } & \multicolumn{2}{|c|}{ NA } \\
\hline MLN present (n) & 2 & 4 & 0 & 0 & 0 & 0 \\
\hline absent (n) & 1 & 15 & 4 & 11 & 2 & 7 \\
\hline $\mathrm{p}$ value & \multicolumn{2}{|c|}{0.169} & \multicolumn{2}{|c|}{ NA } & \multicolumn{2}{|c|}{ NA } \\
\hline LVI present (n) & 0 & 3 & 0 & 0 & 0 & 3 \\
\hline absent (n) & 3 & 16 & 4 & 11 & 2 & 4 \\
\hline $\mathrm{p}$ value & \multicolumn{2}{|c|}{1.000} & \multicolumn{2}{|c|}{ NA } & \multicolumn{2}{|c|}{0.500} \\
\hline
\end{tabular}

EI: extra-thyroidal invasion; MLN: metastatic lymph node; LVI: lymphatic/vascular invasion; NA: not applicable; CPC: conventional papillary carcinoma; PCFV: papillary carcinoma follicular variant; MIFC: minimally invasive follicular carcinoma

*Well differentiated carcinoma-not otherwise specified, poorly differentiated carcinoma and anaplastic carcinoma are not included, because of the limited number of cases 
for tumors in the grey zone between FA and MIFC showing some suspicious but not diagnostic features of capsular and/or vascular invasion and as 'well-differentiated tumor of uncertain malignant potential' in between FA and encapsulated PCFV for encapsulated follicular patterned tumors showing intermediate nuclear features of PC. The antibodies currently used for the differential diagnosis of these follicular patterned lesions sometimes can be very helpful. However, this is not always the case and, not very infrequently, the immunohistochemical findings do not support the morphological features, meaning that the issue remains unsolved. The most preferred way is using a panel of 3 or 4 markers for PC. The most widely used markers are CK19, Galectin-3, HBME-1 and CITED 1. However, none of these markers are highly specific and sensitive alone (1-6).

Pathologists require more specific markers for well differentiated thyroid carcinoma, especially for PCFV.

The discovery of a marker of malignancy is the final goal of the researches in many sections of pathology. For the thyroid, such a marker is required not only in histopathology, but also in cytopathology. Although fine needle aspiration cytology of the thyroid is a well-established diagnostic step in thyroid lesions, it has limitations for distinguishing FA from FC and sometimes FA from NH (adenomatoid nodules). A marker of malignancy would also serve as a definite preoperative diagnostic tool for well differentiated carcinoma by using immunocytochemistry. In this situation, unnecessary surgical operations would be avoided and planning of the extent of the surgery and supplementary treatments would be possible before operating on thyroid carcinoma.

IMP3 appeared to be a promising marker for the diagnosis of malignant tumors of many organs, as well as a potential therapeutic target (29). It is postulated that it might be a biomarker of malignancy (30). In the examined literature, we have only found a few studies evaluating IMP3 expression in thyroid tumors. According to the results of these studies, IMP3 seemed to be a "miracle" marker of malignancy in thyroid, with almost 100\% specificity and PPV (7-9).

Slosar et al. evaluated IMP3 expression in 219 benign and malignant thyroid lesions by using immunohistochemistry (7). They did not observe positive staining in normal thyroid tissue and benign lesions; thus, the specificity and positive predictive value of IMP 3 was both $100 \%$ for FA compared to MIFC and for FA compared to PCFV. However, the sensitivity was not as high as the specificity. The sensitivity of IMP3 was $69 \%$ for FC and 38\% for PCFV. They also made the interesting observation that CPC showed rare and weak staining, whereas PCFV at times showed intense staining; they suggested a common developmental pathway for FC and the PCFV (7).

Jin et al. evaluated the IMP3 expression in thyroid neoplasms by using 2 techniques: immunohistohemistry and qRT PCR. They performed immunohistochemistry in 32 cases. They obtained over $60 \%$ positivity in malignant tumors, while only 1 case of follicular adenoma out of 9 cases showed positive immunoreactivity. They also evaluated 80 cases by qRT PCR and IMP3 expression was detected in all of the thyroid lesions. However, the expression was significantly higher in carcinomas, whereas the relative IMP3 expression was slightly higher in benign lesions than the normal thyroid RNA. IMP3 qRT PCR analysis demonstrated $91.4 \%$ specificity and $86.7 \%$ sensitivity for the diagnosis of well differentiated thyroid carcinoma (8).

Another study in the literature evaluating IMP3 expression in thyroid lesions is that of Asioli et al., investigating the effect of IMP3 expression on prognosis in 56 poorly differentiated thyroid carcinomas. They applied both immunohistochemistry and qRT PCR, and for both techniques, they concluded that the expression of IMP3 seems to be a bad prognostic sign for PDC (9).

In contrast with the previously mentioned studies, we observed IMP3 expression in benign lesions both in NH and FA as well as in carcinomas. Of the $22 \mathrm{NH}$ cases, $11(50 \%)$ of them showed positivity. The positive cases were either IHC score 3 ( 7 cases) or IHC score 4 (4 cases). None of the $\mathrm{NH}$ cases were IHC score 5 or 6 . On the other hand, of the $14 \mathrm{FA}$ cases, $13(92.9 \%)$ were positive and 4 cases were IHC score 5 , while 5 cases were IHC score 6 . Statistically, there was a significant difference between the 2 groups in terms of positive staining $(\mathrm{p}=0.011)$ and the median IHC score $(\mathrm{p}=0.000)$.

Malignant tumors showed $82.1 \%$ positivity overall. There was no significant difference between benign and malignant tumors. Also, when compared with each other, the types and subtypes of malignant tumors had an extent of staining that was not significantly different. We wanted to compare the follicular patterned tumors, the most challenging group, but IMP3 expression again did not differ significantly from each other.

Our results are not compatible with the results and the thesis of previous studies. We obtained a higher frequency of positivity in both benign and malignant lesions. One of the reasons for this could be technical variations, different antibodies, clones, etc. A well-known fact in immunohistochemistry is that sometimes the specificity and sensitivity of the antibodies can show variations with different clones and brands.

Our data suggest that IMP3 is effective for discriminating $\mathrm{NH}$ from neoplastic lesions and that this might be useful information, especially in cytological preparations.

Since there is a limited number of studies evaluating IMP3 expression in thyroid lesions, it is obvious that more data should be gathered to prove the usefulness of IMP3 expression in thyroid lesions.

Ethics Committee Approval: Ethics committee approval was received for this study from the ethics committee of Ankara Numune Hospital (E-14-235).

Peer-review: Externally peer-reviewed. 
Author contributions: Concept - S.K. , G.E.; Design - S.K., G.E.; Supervision - S.K.; Resource - S.K., G.E.; Materials - S.K., G.E.; Data Collection\&/or Processing - S.K., G.E.; Analysis\&/or Interpretation - S.K., G.E.; Literature Search - S.K., G.E.; Writing - S.K.; Critical Reviews - S.K., G.E.

Conflict of Interest: No conflict of interest was declared by the authors.

Financial Disclosure: This study has receieved financial support from Ankara Numune Hospital Scientific Research Support Fund .

\section{REFERENCES}

1. de Matos PS, Ferreira AP, de Oliveira Facuri F, Assumpção LV, Metze K, Ward LS. Usefulness of HBME-1, cytokeratin 19 and galectin-3 immunostaining in the diagnosis of thyroid malignancy. Histopathology 2005;47:391-401. [CrossRef]

2. Scognamiglio T, Hyjek E, Kao J, Chen YT. Diagnostic usefulness of HBME1, galectin-3, CK19, and CITED1 and evaluation of their expression in encapsulated lesions with questionable features of papillary thyroid carcinoma. Am J Clin Pathol 2006;126:700-8. [CrossRef]

3. de Matos LL, Del Giglio AB, Matsubayashi CO, de Lima Farah M, Del Giglio A, da Silva Pinhal MA. Expression of CK-19, galectin-3 and HBME-1 in the differentiation of thyroid lesions: systematic review and diagnostic meta-analysis. Diagn Pathol 2012;7:97. [CrossRef]

4. Ito Y, Yoshida H, Tomoda C, Miya A, Kobayashi K, Matsuzuka F, et al. Galectin-3 expression in follicular tumours: an immunohistochemical study of its use as a marker of follicular carcinoma. Pathology 2005;37:296-8. [CrossRef]

5. Mehrotra P, Okpokam A, Bouhaidar R, Johnson SJ, Wilson JA, Davies BR, et al. Galectin-3 does not reliably distinguish benign from malignant thyroid neoplasms. Histopathology 2004;45:493-500. [CrossRef]

6. Sahoo S, Hoda SA, Rosai J, DeLellis RA. Cytokeratin 19 immunoreactivity in the diagnosis of papillary thyroid carcinoma: a note of caution. Am J Clin Pathol 2001;116:696-702. [CrossRef]

7. Slosar M, Vohra P, Prasad M, Fischer A, Quinlan R, Khan A, et al. Insulin-like growth factor mRNA Binding protein 3 (IMP3) is differentially expressed in benign and malignant follicular patterned thyroid tumors. Endocr Pathol 2009;20:149-57. [CrossRef]

8. Jin L, Seys AR, Zhang S, Erickson-Johnson MR, Roth CW, Evers BR, et al. Diagnostic utility of IMP3 expression in thyroid neoplasms. A quantitative RT-PCR study. Diag Mol Pathol 2009;19:63-9. [CrossRef]

9. Asioli S, Erickson LA, Righi A, Jin L, Volante M, Jenkins S, et al. Poorly differentiated carcinoma of the thyroid: validation of the Turin proposal and analysis of IMP3 expression. Mod Pathol 2010;23:1269-78. [CrossRef]

10. Nielsen J, Christiansen J, Lykke-Andersen J, Johnsen AH, Wewer UM, Nielsen FC. A family of insulin-like growth factor II mRNA-binding proteins represses translation in late development. Mol Cell Biol 1999;19:1262-70.

11. Nielsen FC, Nielsen J, Christiansen J. A family of IGF-II mRNA binding proteins (IMP) involved in RNA trafficking. Scand $J$ Clin Lab Invest Suppl 2001;234:93-9. [CrossRef]

12. Mueller-Pillasch F, Pohl B, Wilda M, Lacher U, Beil M, Wallrapp $\mathrm{C}$, et al. Expression of the highly conserved RNA binding protein KOC in embryogenesis. Mech Dev 1999;88:95-9. [CrossRef]

13. Jiang Z, Chu PG, Woda BA, Rock KL, Liu Q, Hsieh CC, et al. Analysis of RNA-binding protein IMP3 to predict metasta- sis and prognosis of renal-cell carcinoma: a retrospective study. Lancet Oncol 2006;7:556-64. [CrossRef]

14. Jeng YM, Chang CC, Hu FC, Chou HY, Kao HL, Wang TH, et al. RNA-binding protein insulin-like growth factor II mRNAbinding protein 3 expression promotes tumor invasion and predicts early recurrence and poor prognosis in hepatocellular carcinoma. Hepatology 2008;48:1118-27. [CrossRef]

15. Li C, Zota V, Woda BA, Rock KL, Fraire AE, Jiang Z, et al. Expression of a novel oncofetal mRNA-binding protein IMP3 in endometrial carcinomas: diagnostic significance and clinicopathologic correlations. Mod Pathol 2007;20:1263-8. [CrossRef]

16. Li C, Rock KL, Woda BA, Jiang Z, Fraire AE, Dresser K, et al. IMP3 is a novel biomarker for adenocarcinoma in situ of the uterine cervix: an immunohistochemical study in comparison with p16 (INK4a) expression. Mod Pathol 2007;20:242-7. [CrossRef]

17. Kobel M, Xu H, Bourne PA, Spaulding BO, Shih IeM, Mao TL, et al. IGF2BP3 (IMP3) expression is a marker of unfavorable prognosis in ovarian carcinoma of clear cell subtype. Mod Pathol 2009;22:469-75. [CrossRef]

18. Samanta S, Sharma VM, Khan A, Mercurio AM. Regulation of IMP3 by EGFR signaling and repression by ER $\beta$ : implications for triple-negative breast cancer. Oncogene 2012;31:4689-97. [CrossRef]

19. Sidoni A, Cartaginese F. IMP3 expression in triple-negative breast carcinoma. Hum Pathol 2010;41:1355-6. [CrossRef]

20. Yantiss RK, Woda BA, Fanger GR, Kalos M, Whalen GF, Tada $\mathrm{H}$, et al. KOC ( $\mathrm{K}$ homology domain containing protein overexpression in cancer): a novel molecular marker that distinguishes between benign and malignant lesions of the pancreas. Am J Surg Pathol 2005;29:188-195. [CrossRef]

21. Wang L, Li HG, Xia ZS, Lü J, Peng TS. IMP3 is a novel biomarker to predict metastasis and prognosis of gastric adenocarcinoma: a retrospective study. Chin Med J (Engl) 2010;123:3554-8.

22. Li D, Yan D, Tang H, Zhou C, Fan J, Li S, et al. IMP3 is a novel prognostic marker that correlates with colon cancer progression and pathogenesis. Ann Surg Oncol 2009;16:3499-506. [CrossRef]

23. Sitnikova L, Mendese G, Liu Q, Woda BA, Lu D, Dresser K, et al. IMP3 predicts aggressive superficial urothelial carcinoma of the bladder. Clin Cancer Res 2008;14:1701-6. [CrossRef]

24. Beljan Perak R, Durdov MG, Capkun V, Ivcevic V, Pavlovic A, Soljic V, et al. IMP3 can predict aggressive behaviour of lung adenocarcinoma. Diagn Pathol 2012;7:165. [CrossRef]

25. Xu H. IMP3: a diagnostic and prognostic biomarker in malignant melanoma. Expert Rev Mol Diagn 2008;8:557-8. [CrossRef]

26. Do SI, Kim YW, Park HR, Park YK. Expression of insulin-like growth factor-II mRNA binding protein 3 (IMP3) in osteosarcoma. Oncol Res 2008;17:269-72. [CrossRef]

27. Cornejo K, Shi M, Jiang Z. Oncofetal protein IMP3: a useful diagnostic biomarker for leiomyosarcoma. Hum Pathol 2012;43:1567-72. [CrossRef]

28. Liao B, Hu Y, Herrick DJ, Brewer G. The RNA-binding protein IMP-3 is a translational activator of insulin-like growth factor II leader-3 mRNA during proliferation of human K562 leukemia cells. J Biol Chem 2005;280:18517-24. [CrossRef]

29. Mueller F, Bommer M, Lacher U, Ruhland C, Stagge V, Adler $\mathrm{G}$,et al. KOC is a novel molecular indicator of malignancy. $\mathrm{Br} J$ Cancer 2003;88:699-701. [CrossRef]

30. Kapoor S. IMP3: a new and important biomarker of systemic malignancies. Clin Cancer Res 2008;14:5640. [CrossRef] 